\title{
S-Transform Based Time-Frequency Analysis of Leakage Current Signals of Transmission Line Insulators under Polluted Conditions
}

\author{
A. Natarajan ${ }^{\dagger}$ and Suthanthiravanitha Narayanan*
}

\begin{abstract}
Flashover of power transmission line insulators due to contamination is a major threat to the reliable operation of power system. This paper deals with the analysis of leakage current characteristics of polymeric insulator using S-Transform technique in order to develop a better diagnostic tool to identify the surface condition of outdoor polymeric insulators. In this work, experiments were carried out on $11 \mathrm{kV}$ silicone rubber insulator under AC voltage at different pollution levels. Moving average technique was adopted to find the trend followed by LC peak at different relative humidity conditions. S-Transform was used to find the relationship between energy and frequency content of the leakage current signal with respect to increase in pollution level over a period of time. From the S-Transform time-frequency contour analysis, point of transition to severe arcing due to increase in pollution and its thershold limit were evaluated. Reported results show that the surface condition of insulators could be easily identified from the S-Transform time-frequency analysis of leakage current signals.
\end{abstract}

Keywords: Silicone rubber, Insulator, Flashover, S-Transform, Pollution, Time-frequency map

\section{Introduction}

Ceramic and polymeric insulators are widely used in power transmission and distribution systems. In recent times, silicone rubber polymeric insulators are mostly preferred because of their superior insulation and surface hydrophobicity characteristics compared with other insulators $[1,2]$. The hydrophobicity of silicone rubber material provides high electrical surface resistance. The long term maintenance of the hydrophobicity of silicone rubber insulator is mainly due to its chemical stability and diffusion of low molecular weight contents from bulk volume to the surface of the material [3]. However, when these insulators are installed near industrial, agricultural or coastal areas, pollution builds up gradually on the surface of the insulator, which results in the flow of leakage current (LC) under wet conditions and finally leads to arcing and flashover. Contamination flashover of outdoor insulators results in power outages, waste of time and money and sometimes equipment damage. In addition, continuous arcing on the surface of the insulator results in material degradation and reduction in hydrophobicity, which in turn decreases the insulation strength. Therefore electrical utilities are keen in the development of a better diagnostic tool to predict exactly the surface condition of polymeric insulators under severe pollution conditions.

Analysis of surface deterioration of the polymeric

$\dagger$ Corresponding Author: Dept. of Electronics and Communication Engineering, Annai Mathammal Sheela Engineering College, Namakkal. India. (anatarajanrsp@gmail.com)

* Dept. of Electrical and Electronics Engineering, Knowledge Institute of Technology, Salem, India. (varmans03@gmail.com)

Received: May 16, 2014; Accepted: October 13, 2014 insulators due to the formation of surface discharges is a complex process. Various approaches were used to predict the flashover and surface degradation of outdoor insulators [4-17]. R.S.Gorur et al., [7] measured the surface resistance to identify the surface condition of non-ceramic insulators. Many research papers deal with the measurement and analysis of LC, because it is directly related to arcing phenomena occurring on insulator [8-11]. S.Kumagai et al., [13] have compared the LC characteristics and aging of porcelain and polymeric insulator in both field and salt fog tests. They have concluded that the time variations of cumulative charges and their component ratios were useful for estimating the conditions of ceramic and polymeric insulating surfaces.

From the reported earlier investigations, it is clear that LC follows different pattern during the deveopment of flashover, from lightly polluted conditions to heavily polluted conditions. L.H.Meyer et al., [14] have shown that there exists a good correlation between the measured harmonic power components of dry band arcing and the surface temperature of silicone rubber samples. T.Suda [15], studied the LC waveforms and frequency characteristics of an artificially polluted cap and pin type insulator and classified the transition of LC waveforms into six stages in order to predict the flashover. Proper understanding of the various LC pattern and its time-frequency characteristics is very important in order to evaluate the surface condition of polymeric insulators.

M.Ugur et al., [16] proposed a methodology to classify the surface condition of the polymeric insulation structure using neural network approach by utilizing the features extracted from the FFT analysis of the LC during tracking 
test. FFT is most suited for stationary time series signals. In the case of transmission line insulators, LC signal is a nonstationary time series signal, in which spectral content changes with time and hence Fourier Transform is inadequate to track the changes in the LC signal magnitude, frequency or phase. S.Chandrasekar et al., [17] applied the discrete wavelet transform to identify the surface condition of the silicone rubber insulating material under different pollution conditions. However, major drawback of wavelet analysis is the selection of proper mother wavelet in order to identify the best one for a better classification. In addition, eventhough discrete wavelet transform provides good time-frequency resolution, the information is sparse and the spectral regions are not unique and different dilations have overlapping spectral responses.

Considering the importance of developing a better diagnostic tool to exactly predict the point of transition to severe arcing of outdoor insulators, in the present work, an advanced signal processing technique, S-Transform, has been adopted. S-transform produces the time-frequency representation of a time domain signal. It uniquely combines a frequency-dependent resolution that simultaneously localizes the real and imaginary spectra [18-19]. S-transform overcomes the above mentioned drawbacks of FFT and DWT and is well suited for transient analysis of signals under noisy environment. It has good localization in both time and frequency domain, which makes it suitable for time-frequency analysis.

In the present work, a methodology to monitor the surface condition of the polymeric insulating material using $\mathrm{S}$-transform is proposed. LC measurements are carried out in the laboratory on silicone rubber insulator at different pollution levels. Energy content and time-frequency contour characteristics of LC signal are evaluated using S-Transform and point of transition to severe arcing is identified.

\section{Test Setup and LC Measurement System}

Commercially available $11 \mathrm{kV}$ polymeric insulators with specifications as listed in Table 1 were used for laboratory experiments. Tests were conducted as per IEC 60507 test procedures [20]. Fig. 1 shows the photograph and dimensions of polymeric insulator used in this study.

Fig. 2 shows the schematic diagram of the experimental setup, where PU-Protection Unit, DAS-Data Acquisition System, DSO-Digital Storage Oscilloscope, PC-Personal Computer, UN- Ultrasonic nebulizer, HTM- Hygrotherm meter. Test insulator was suspended vertically inside the fog chamber $(1.5 \times 1.5 \times 1.5 \mathrm{~m})$ with necessary protections. A high voltage transformer of $100 \mathrm{kV}, 10 \mathrm{kVA}$ capacity was used to supply the required voltage. Contamination solution was prepared with $\mathrm{NaCl}(10 \mathrm{~g}$ to $100 \mathrm{~g})$ and $40 \mathrm{~g}$ of kaolin mixed with 1 litre of deionized water. Insulator specimen was applied with $11 \mathrm{kVrms}$. Four ultrasonic nebulizers were used to maintain the required relative

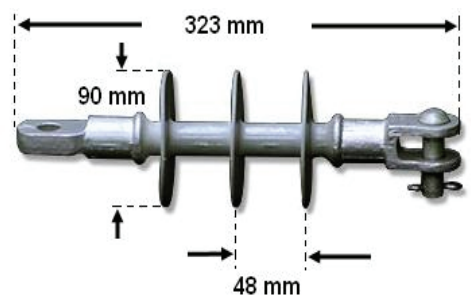

Fig. 1. Dimensions of the $11 \mathrm{kV}$ silicone rubber insulator

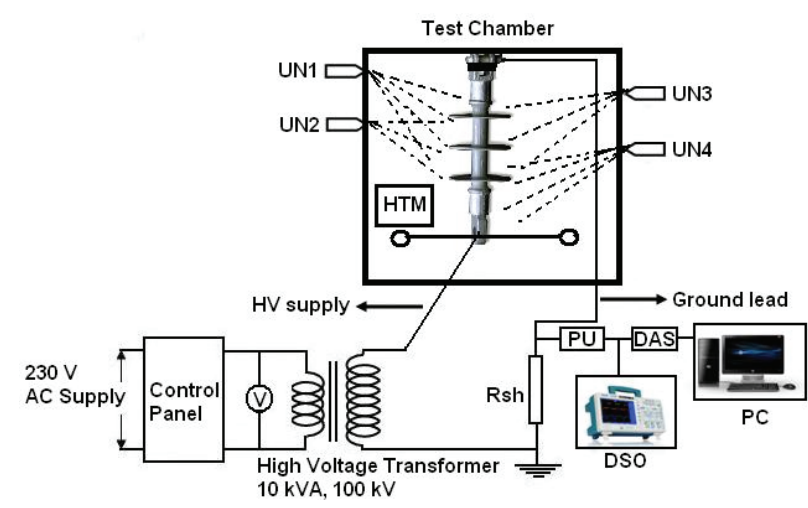

Fig. 2. Schematic diagram of the experimental set up

humidity level inside the fog chamber. and its value was measured using the wall-mount Hygrotherm meter. Before test, the insulator was cleaned with isopropylic alcohol and rinsed with distilled water, in order to remove any trace of dirt and grease.

LC was measured through the shunt resistance connected in the ground lead. A high sampling rate data acquisition system (National Instruments USB 6251, $1.25 \mathrm{MSa} / \mathrm{sec}$ ) was used to capture the signals at a sampling rate of $5 \mathrm{kHz}$ and the data was stored in $\mathrm{PC}$ for further processing. A software system developed for this data acquisition system provides the user with the complete LC waveforms, which are therefore available for further signal processing. A Digital Storage Oscilloscope $(1 \mathrm{GSa} / \mathrm{sec})$ was used to visualize the LC patterns.

\section{Concept of S-Transform}

The S-transform is similar to the wavelet transform but with a phase correction. The S-transform of a time series functon $h(t)$ can be obtained by multiplying the continuous wavelet transform with a phase factor. It is a time frequency spectral localization method, similar to Short Time Fourier transform (STFT), but with a Gaussian window whose width scales inversely and height linearly with the frequency [18-19]. The expression for the Stransform is given as,

$$
S(\tau, f)=e^{-i 2 \pi f \tau} W(\tau, d)
$$

where $\tau$ is the translation parameter, $f$ is frequency and $d$ is 
the scale parameter. $W(\tau, d)$ is the continuous wavelet transform of function $h(t)$. The phase factor in Eq. (1) is a phase correction introduced to the wavelet transform.

The discrete version of the S-transform is calculated by taking the advantage of the efficiency of the Fast Fourier Transform (FFT) and the convolution theorem. The discrete Fourier transform of the sampled signal $h(k T), \mathrm{k}=0$, $1, \mathrm{~N}-1$ is,

$$
H\left(\frac{n}{N T}\right)=\frac{1}{N} \sum_{k=0}^{N-1} h(k T) e^{-i(2 \pi n k / N)}
$$

and discrete version of the S-transform of $\mathrm{h}(\mathrm{kT})$ is obtained as (by letting $f \rightarrow n / N T$ and $\tau \rightarrow j T$ )

$$
S\left(j T, \frac{n}{N T}\right)=\sum_{m=0}^{N-1} H\left(\frac{m+n}{N T}\right) G(m, n) e^{i\left(\frac{2 \pi m j}{N}\right)}
$$

where

$$
G(m, n)=e^{-\left(2 \pi^{2} m^{2} \alpha^{2} / n^{2}\right)}
$$

$j, m$, and $\mathrm{n}=0,1,2, \ldots . . N-1$, and $\mathrm{N}=$ total number of samples.

The discrete inverse of S-transform is obtained as

$$
h(k T)=\frac{1}{N} \sum_{n=o}^{N-1}\left\{\sum_{j=0}^{N-1} S\left[\frac{n}{N T}, j T\right]\right\} e^{i(2 \pi n k / N)}
$$

It can be noted that the S-transform improves the STFT in that it has a better resolution in phase space giving a fundamentally more sound time-frequency representation.

\section{Flashover Development Process}

The un-aged silicone rubber insulating materials are generally hydrophobic in nature. However, during service the insulator surface gets aged due to contamination from coastal areas and industries. In addition, thermal stress, UV stress, water absorption stress and operating electrical stress acts on the surface of the polymeric insulating material and thereby the material slowly loses its hydrophobicity. Under wet conditions, formation of contamination layer allows the flow of LC and causes partial evaporation of the contaminant due to the heating effect, which leads to the formation of dry bands [10, 12]. This causes voltage redistribution along the insulator

Table 1. Insulator specifications

\begin{tabular}{c|c}
\hline Creepage distance & $307 \mathrm{~mm}$ \\
\hline Shed diameter & $90 \mathrm{~mm}$ \\
\hline Wet power frequency withstand voltage & $35 \mathrm{kV}$ \\
\hline Dry lightning impulse withstand voltage & $75 \mathrm{kV}$ \\
\hline Rated mechanical tensile load & $45 \mathrm{kN}$ \\
\hline Operating temperature & -100 to $300^{\circ} \mathrm{C}$ \\
\hline
\end{tabular}
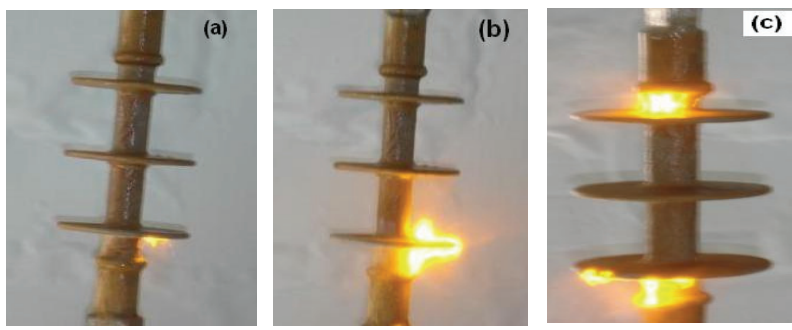

Fig. 3. Photograph of (a) Short duration surface discharges at light pollution; (b) Long arc at medium pollution; (c) Multiple long arcs at heavy pollution.

surface and in turn leads to high electric field intensity across dry bands and formation of partial arcs over the dry band. This partial arc results in a gradual increase in the surface temperature which leads to the formation of carbonization / polymerization / chain scission of polymeric material. Increase in local temperature and reaction of contaminant with the material are considered to be responsible for the surface degradation. Under highly polluted wet conditions, the surface resistance of the material is sufficiently low and these partial discharges will elongate along the insulator profile which may eventually cause the insulator flashover [17]. Fig. 3 shows the photographs of the flashover development process taken during the laboratory experiments from short arcs to long arcs at different pollution levels.

\section{Trend Analysis of LC Peak Magnitude}

Initially, LC signals are captured at a constant $11 \mathrm{kV}$ AC voltage applied to the test specimen at different pollution level with an increasing relative humidity of the fog chamber.

Fig. 4(a) shows a typical trend of raw data of LC peak at high pollution. It is noticed that LC flow is highly intermittent in nature and hence it is difficult to accurately classify the surface condition of the insulator just by performing time-domain analysis of the trend followed by the LC peak. Hence moving average technique is employed to find the trend of LC peak [10]. Figs. 4(b, c, d) shows the moving average trend followed by the LC peak value with increase in relative humidity of the fog chamber at clean surface, $30 \mathrm{~g} / 1 \mathrm{NaCl}$ and $100 \mathrm{~g} / 1 \mathrm{NaCl}$ pollution conditions. In general, considerable increase in LC peak is noticed with respect to increase in pollution level and relative humidity value above $80 \%$. However, presence of large number of peaks and valleys are noticed with respect to increase in pollution level, which makes it difficult to exactly predict the surface condition of insulator at a particular time period. It is necessary to perform both time and frequency domain analysis of LC signals in order to estimate the surface condition of the insulators. Therefore, in the following sections, analysis of LC signals using STransform time-frequency contour pattern is discussed. 

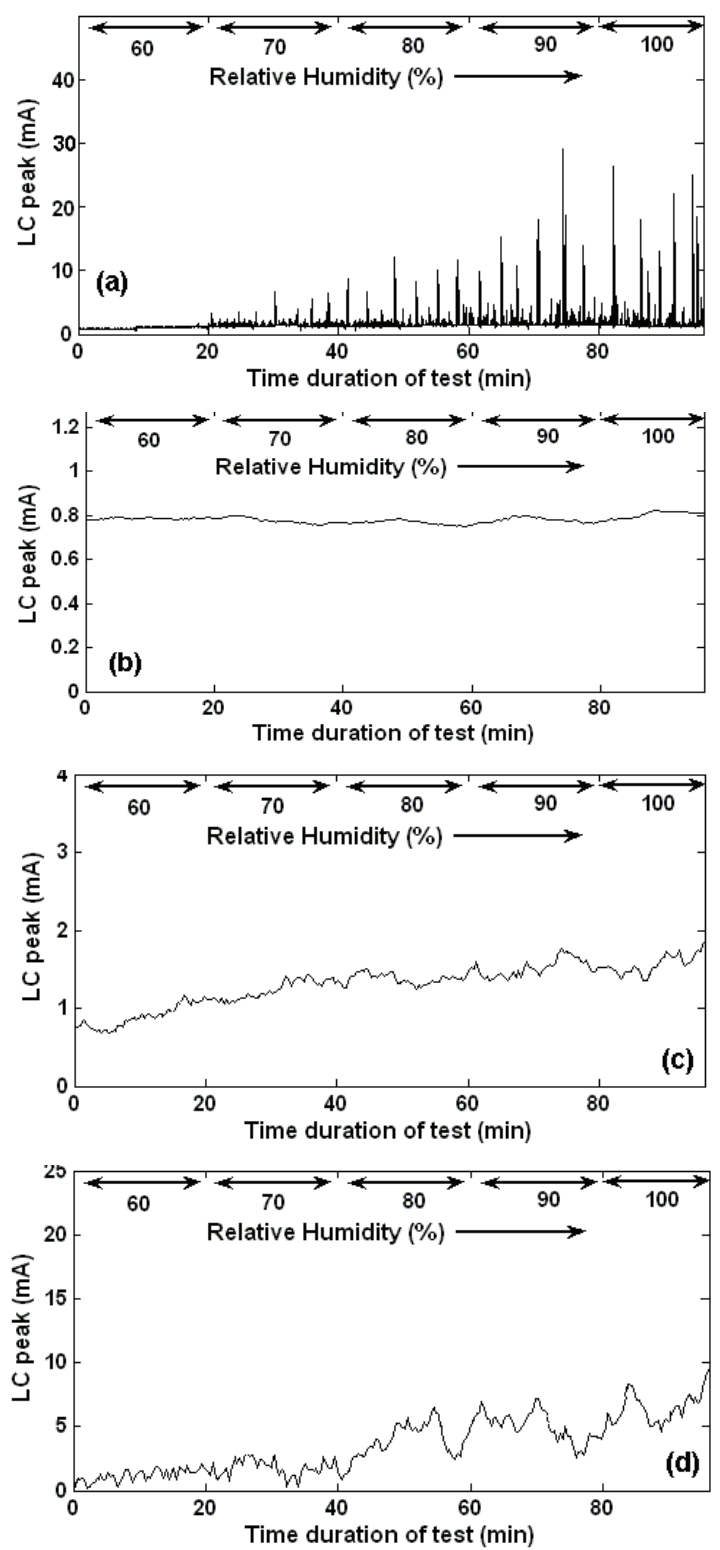

Fig. 4. Trend followed by LC peak: (a) raw data and moving average plot at (b) clean surface (c) $30 \mathrm{~g} / \mathrm{l}$ $\mathrm{NaCl}$ medium pollution (d) $100 \mathrm{~g} / \mathrm{l} \mathrm{NaCl}$ high pollution

\section{S-Transform Analysis of Dry-Clean Insulator}

Initially a silicone rubber insulator was tested inside the fog chamber with an applied voltage of $11 \mathrm{kVrms}$ at dry and clean surface conditions.

Fig. 5(a) shows the typical LC waveform obtained at dry clean condition and it is noted that current magnitude is very low and it is completely distorted. It was noticed that even at high relative humidity level, negligible amount of LC only flows on the surface of the insulator (typically $<0.2 \mathrm{~mA} \mathrm{rms}$ ) due to clean surface condition. Fig. 5 (b) and 5 (c) shows the time-frequency contour plot and 3-D plot respectively obtained using S-Transform
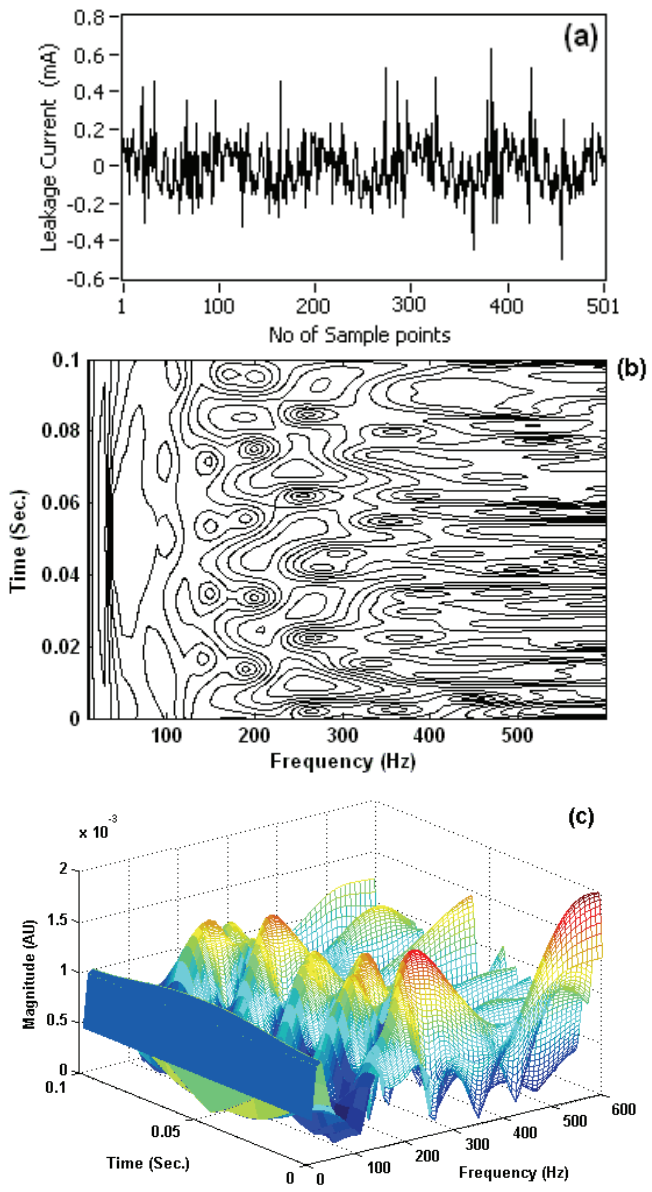

Fig. 5. (a) Typical LC signal during the dry-clean surface

(b) Time-Frequency plot using S-Transform and (c) S-Transform 3-D plot

approach. MATLAB software was used to perform STransform analysis of LC signals. From the S-Transform contour plot and $3 \mathrm{D}$ plot, it is clear that high frequency components (above $50 \mathrm{~Hz}$ ) with a larger magnitude are present in the signal. It is also observed that the LC signal contains higher order harmonic components in which magnitude of $5^{\text {th }}, 7^{\text {th }}, 9^{\text {th }}$ and $11^{\text {th }}$ are higher when compared with $3^{\text {rd }}$ order harmonic components. Magnitude of fundamental component is less when compared with high frequency components.

\section{S-Transform Analysis of Wet-Polluted Insulator}

\subsection{Initial leakage current at lightly polluted surface}

In this test, insulators are tested with increasing pollution level. Outdoor insulators located in coastal areas are mostly affected due to the deposition of $\mathrm{NaCl}$ salt particles. Therefore, initially the silicone rubber insulator was sprayed with $10 \mathrm{~g} / \mathrm{l} \mathrm{NaCl}$ light contamination solution and the relative humidity of the fog chamber was maintained 

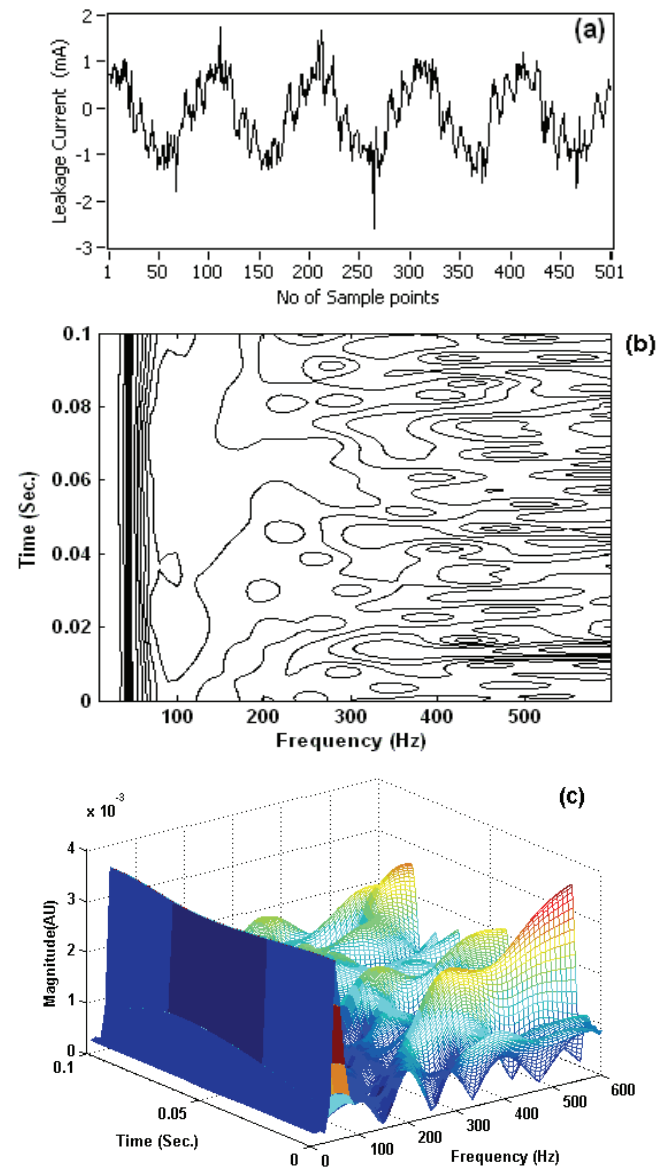

Fig. 6. (a) Typical LC pattern obtained during the wetlightly polluted surface; (b) Time-Frequency plot using S-Transform and (c) corresponding 3-D plot

above $95 \%$. When the rated voltage is applied, leakage current started flowing on the surface of the insulator due to pollution and high relative humidity. Fig. 6 (a) shows the initial leakage current flow under wet conditions when the pollution layer builds up over the surface of the insulator. In this condition, no visible discharge is observed. The time-frequency contour obtained using Stransform approach and corresponding 3D plot is shown in Fig.6 (b) and (c).

It is observed that there is slight increase in LC magnitude when compared with dry-clean surface condition, which is mainly because of increase in surface conductivity due to wet pollution. In the S-transform contour plot, a thick dark line is now formed corresponding to $50 \mathrm{~Hz}$, showing that the magnitude of fundamental frequency component increases when compared with the higher order harmonic components. Compared with dry clean surface condition, it is clear that density of time-frequency contour in the high frequency region is considerably reduced now $[10,12,17]$.

\subsection{Short duration discharges at medium pollution}

In this test, pollution level is further increased to $30 \mathrm{~g} / 1$
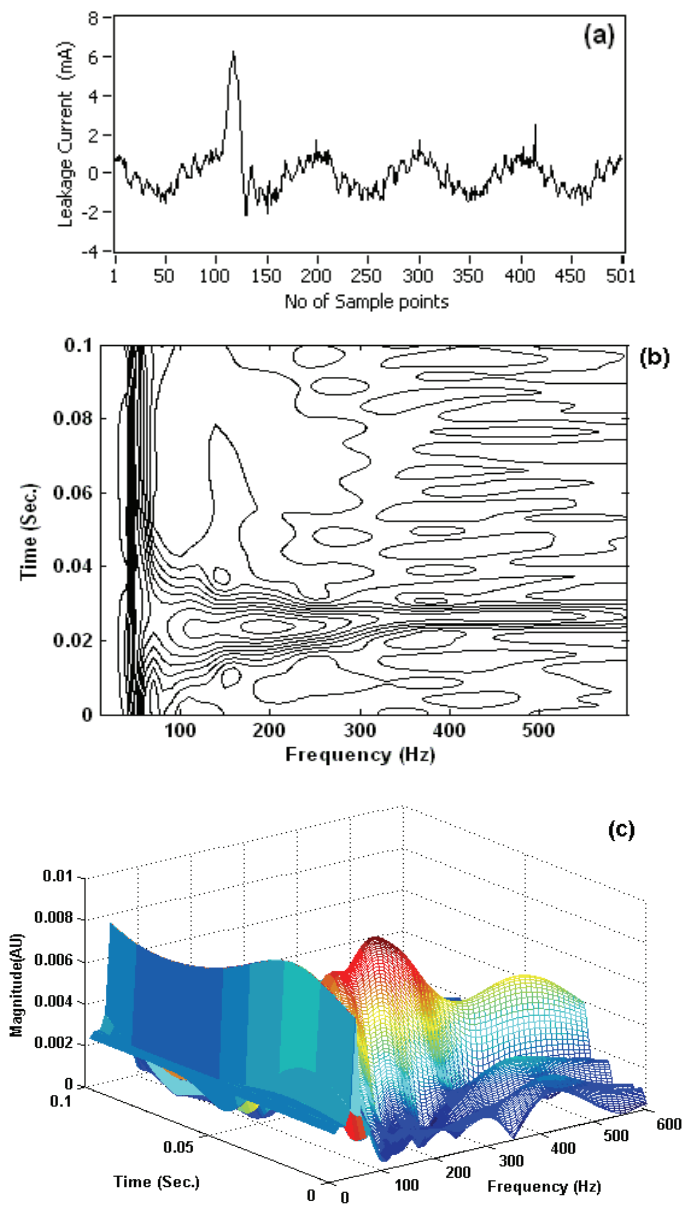

Fig. 7. (a) Typical single short duration discharge obtained at $30 \mathrm{~g} / 1 \mathrm{NaCl}$ wet-polluted surface; (b) TimeFrequency plot using S-Transform and (c) corresponding 3-D plot

of $\mathrm{NaCl}$ and rated voltage is applied under $95 \%$ relative humidity conditions.

From the experimental observations, inception of visual discharges leading to the distortions in initial LC waveform is noticed. Fig. 7(a) shows a typical visual single short duration discharge obtained during the experiment. It is possible to classify the short duration discharges as single (which exist for a maximum of 1 cycle) and repetitive (which exist for 3 to 5 cycles). Fig. 8(a) shows a typical repetitive short duration discharges obtained at this pollution level. These short duration discharges are the precursors for the development of long arcs which will lead to flashover [17]. It is observed that whenever a short duration discharge occurs on the surface then corresponding increase in the magnitude of third harmonic component is noticed in the time-frequency contour as shown in Figs. $7(\mathrm{~b}, \mathrm{c})$ and $8(\mathrm{~b}, \mathrm{c})$. For example in Fig. 7(b), in the time duration 0.02 to $0.04 \mathrm{sec}$, formation of third harmonic component is clearly visible. Similarly, in Fig. 8(b), in the time duration 0.04 to $0.1 \mathrm{sec}$, formation of third harmonic component is clearly visible. 

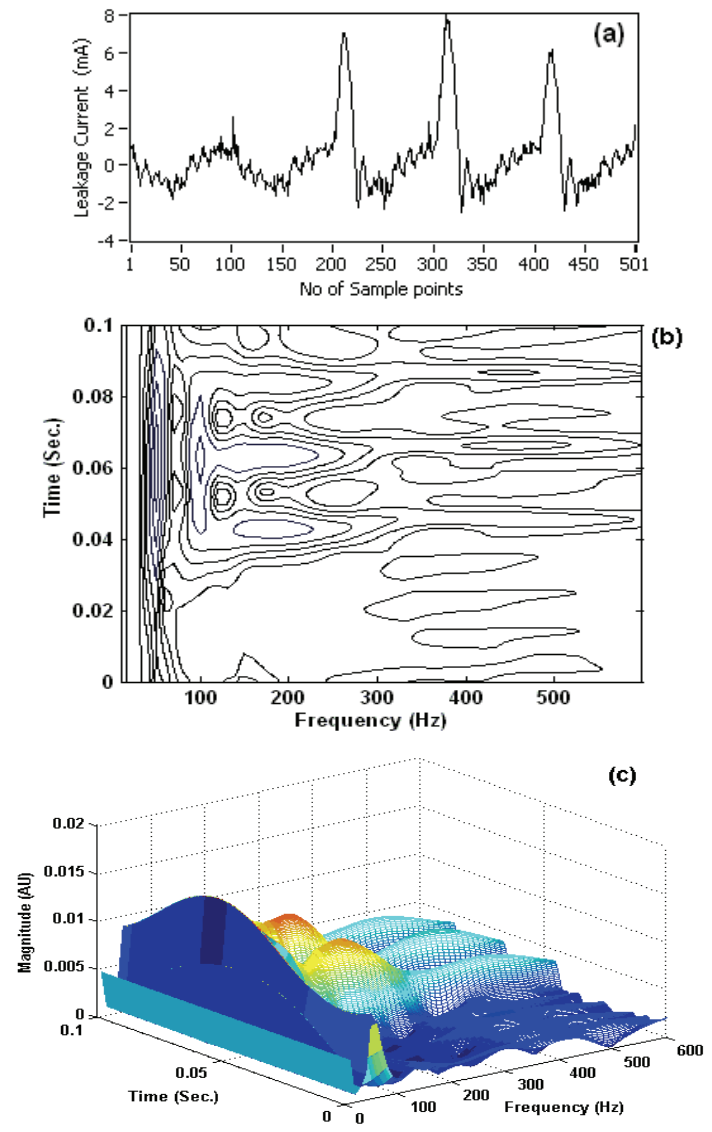

Fig. 8. (a) Typical repetitive short duration discharges obtained at $30 \mathrm{~g} / 1 \mathrm{NaCl}$ wet-polluted surface; (b) Time-Frequency plot using S-Transform and (c) 3D plot

Also from the results, it is noticed that when the frequency of occurrence of short duration discharges increases, then the magnitude of fundamental component also increases along with third harmonic component. Therefore these short duration discharges and corresponding third harmonic component plays a major role in the increase in local surface temperature of the material, which in turn leads to the surface degradation and aging of the material $[10,12]$.

\subsection{Long duration discharges at high pollution}

Silicone rubber insulator was tested with contamination solution consisting of $50 \mathrm{~g} / 1 \mathrm{NaCl}$ (which corresponds to high pollution) at high relative humidity conditions ( $>$ $95 \%$ ). In addition to the large number of short duration discharges, such test conditions also caused the formation of long duration surface discharges at both top and bottom surface. The frequency of occurrence of long duration surface discharges is low when compared with short duration discharges. It is essential to understand the magnitude of long duration surface discharge and its frequency contents in order to give a deeper insight on the surface condition of the polymeric insulators. Magnitude of LC is significantly increased during long duration surface
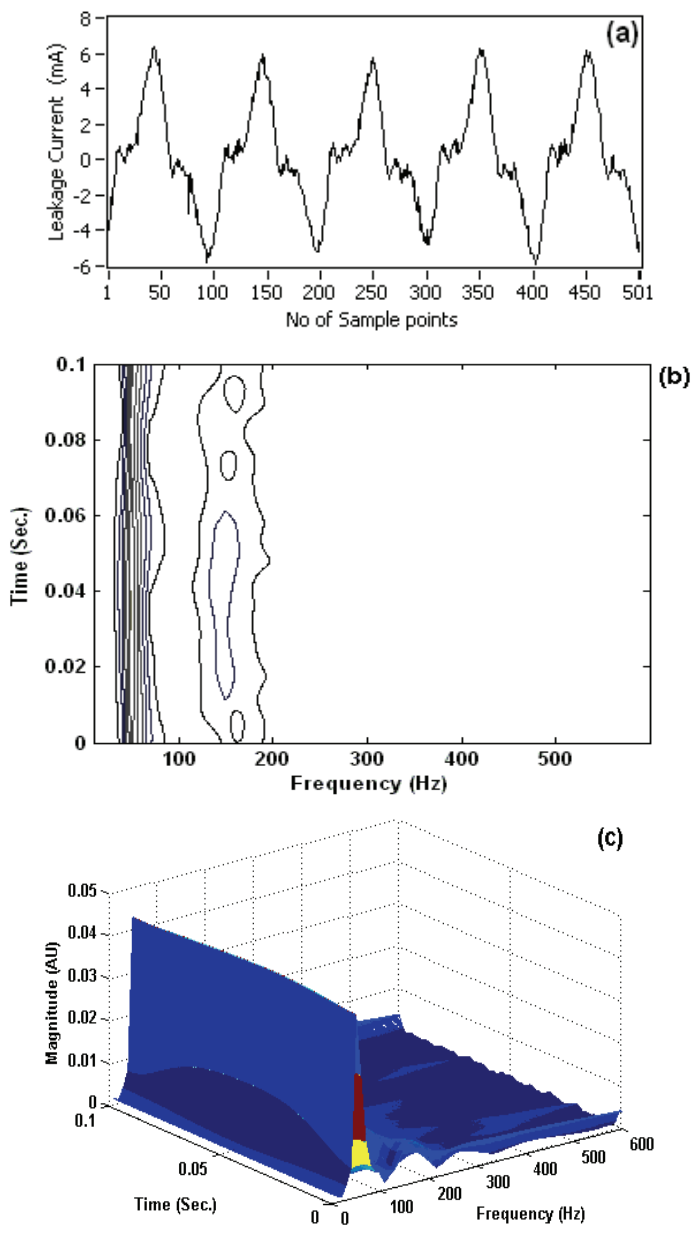

Fig. 9. (a) Typical long duration surface discharge obtained during the $50 \mathrm{~g} / 1 \mathrm{NaCl}$ wet-polluted surface; (b) Time-Frequency plot using S-Transform and (c) corresponding 3-D plot

discharge as shown in Fig. 9(a). From Fig. 9 (b and c), it is observed that the high frequency components $\left(5^{\text {th }}, 7^{\text {th }}, 9^{\text {th }}\right.$, $11^{\text {th }}$ order harmonics) are completely absent during the formation of long duration discharges [17]. Only the presence of third harmonic component is noticed. Increase in magnitude of fundamental component of LC when compared with short duration discharge pattern is also observed from the 3-D plot.

\subsection{Heavy arcing at very high pollution}

When the insulator is tested at very high pollution (100 $\mathrm{g} / \mathrm{l})$, the LC pattern almost looks like sinusoidal waveform with a very high magnitude (Fig. 10 a). From the Stransform time-frequency contour plots (Fig. $10 \mathrm{~b}$ and c), it is observed that most of the high frequency components are completely lost in this phase.

It is clear that the heavy arcing current contains mainly fundamental frequency component with a reduction in third order harmonic contents [17]. In addition, at the time of flashover, predominant increase in the value of 

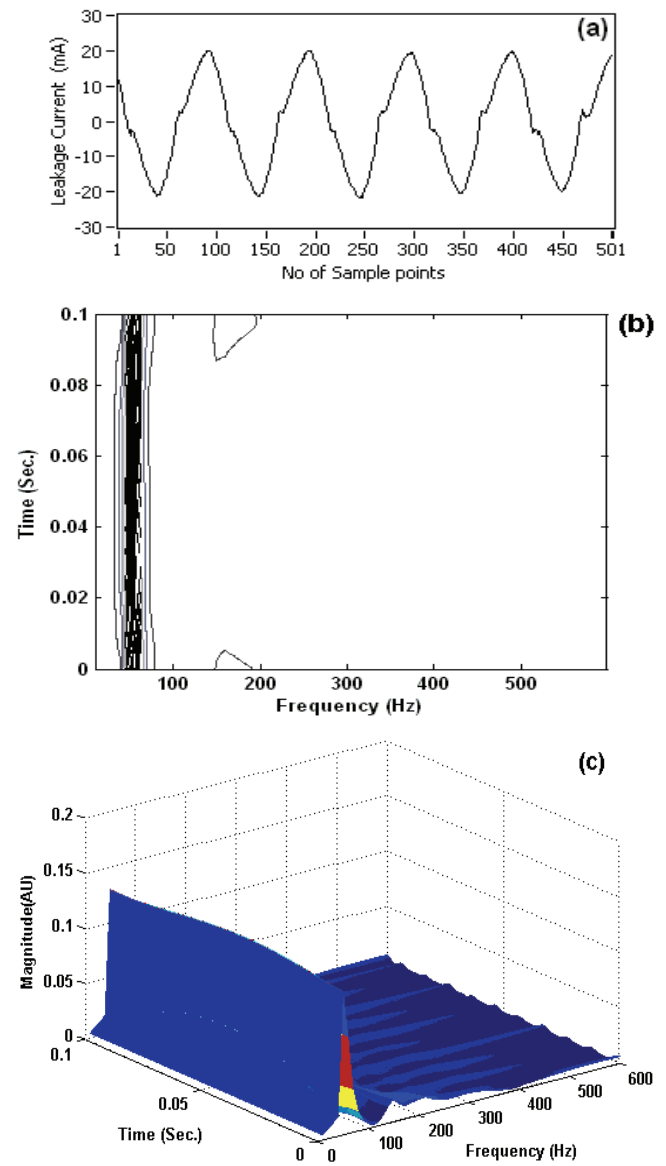

Fig. 10. (a) Typical heavy arcing signal obtained during the $100 \mathrm{~g} / \mathrm{l} \mathrm{NaCl}$ wet-polluted surface: (b) TimeFrequency plot using S-Transform and (c) corresponding 3-D plot

fundamental component of current is noticed when compared with short duration discharge signals.

\subsection{Prediction of point of transition to heavy arcing}

S-transform results clearly shows that it is possible to visually classify the surface condition of the outdoor insulators. However in order to develop a better diagnostic tool to predict exactly the transition of insulator surface from short duration discharge to heavy arcing, it is necessary to extract important features such as energy content and frequency content from the S-Transform timefrequency representation of LC signal. Standard deviation can be considered for the evaluation of energy content of the signal [17]. Fig. 11 shows the relationship between the energy content and frequency content of the LC signal obtained from S-Transform. As discussed in the previous sections, it is clear that energy content of high frequency components are higher at lightly polluted conditions whereas energy content of the fundamental component of signal increases with increase in pollution. It is noticed that the LC signal evolution at every stage of flashover process has distinct energy content-frequency patterns.
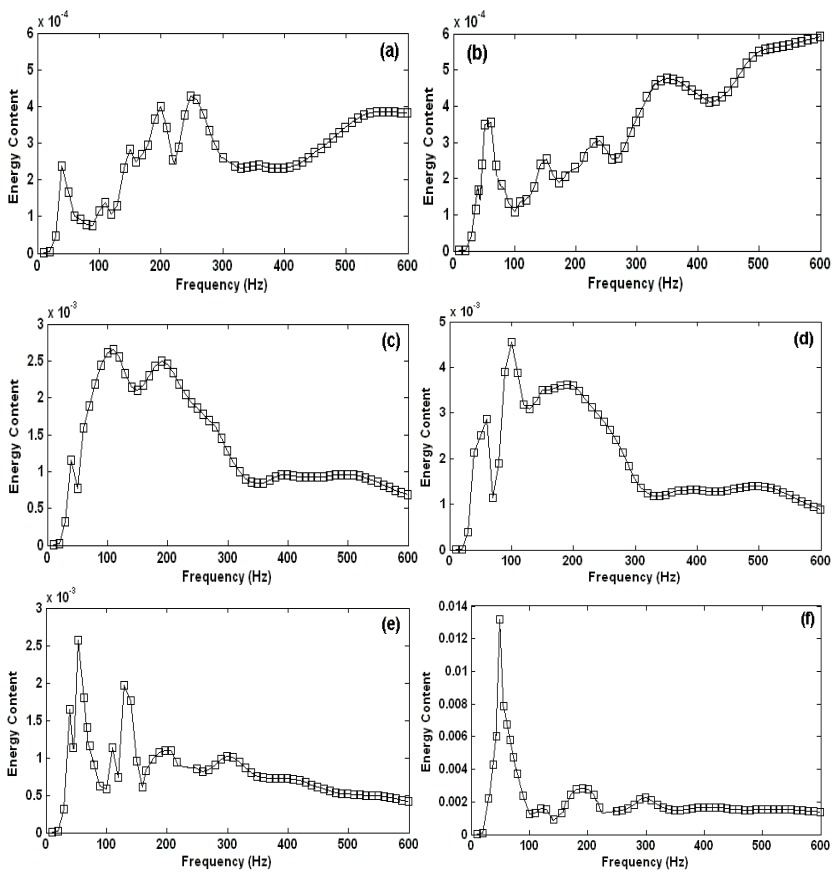

Fig. 11. Energy content of signal derived from S-Transform w.r.t increase in frequency at different surface conditions: (a) dry-clean; (b) wet-clean; (c) short duration single discharge; (d) short duration multiple discharge; (e) long duration surface discharge; (f) heavy arcing
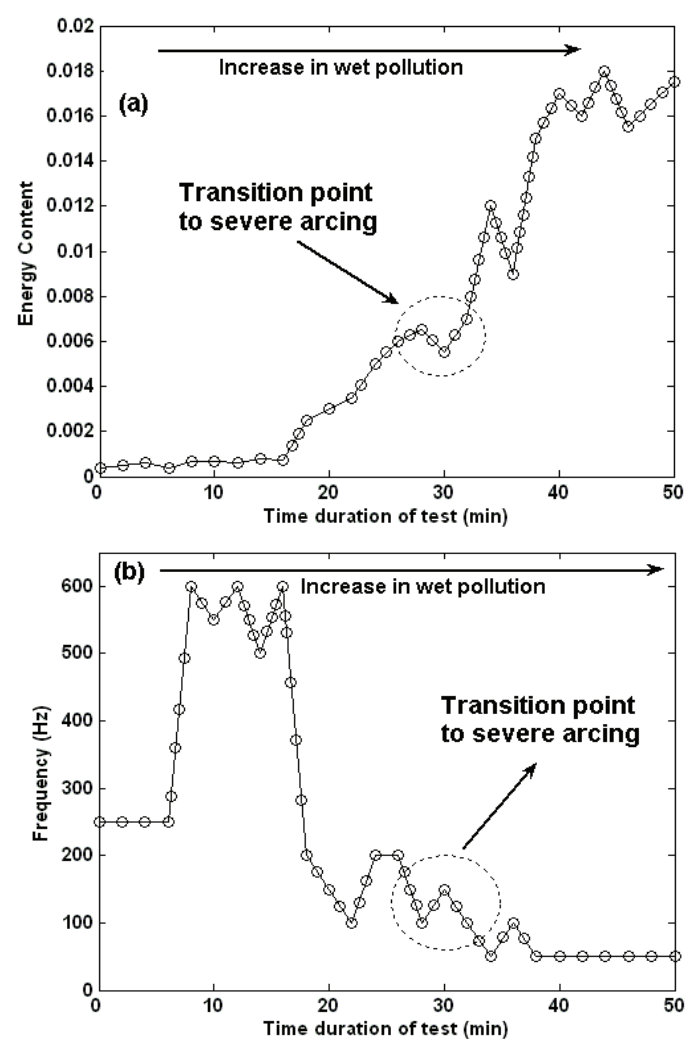

Fig. 12. Variations in (a) energy content and (b) frequency content of LC signal with respect to increase in pollution 
It is observed that energy content and frequency content of the signal has a very good correlation with the surface pollution condition and surface discharges. Hence in the present work, peak value of energy content, corresponding frequency content and its time of occurrence with respect to increase in pollution level of LC signals using the STransform technique are evaluated to exactly predict the transition point to severe arcing. Fig. 12 shows the moving average plot of peak energy content and corresponding frequency content of LC signal with respect to increase in pollution and test duration. It clearly shows the transition point to severe arcing due to very high pollution. Trend analysis of these two features can be considered to set the thershold limit to take necessary preventive measures by the substation operator.

\section{Conclusion}

Experimental results on silicone rubber insulator to understand the pollution severity from LC measurement and its analysis using S-transform technique has been presented in this paper. S-Transform time-frequency contour clearly shows the variations in magnitude and frequency content of LC signal with respect to increase in pollution level. Relationship between energy content and frequency content of LC signal at different pollution levels is evaluated and the point of transition to severe arcing is predicted. These results clearly show that accurate identification of surface condition of the polymeric insulation structure is possible from the S-transform time-frequency contour approach.

\section{Acknowledgement}

Authors would like to sincerely thank the Head of GnanEXPERT R\&D Centre of Gnanamani College of Technology and team members for providing support in the laboratory experimental studies.

\section{References}

[1] R.S. Gorur, E.A. Cherney and J.T. Burnham, Outdoor Insulators, Ravi S. Gorur Inc Phoenix, Arizona 85044, USA, 1999.

[2] Looms J.S.T., Insulators for high voltages, IEE series, 1990.

[3] S.H. Kim, E.A. Cherney and R. Hackam, "Hydrophobic behaviour of Insulators Coated with RTV Silicone Rubber", IEEE Trans. on Electr. Insul., vol. 27, no. 3, pp. 610-622, 1992.

[4] G. Montoya, I. Ramirez, J. I. Montoya, "Correlation among ESDD, NSDD and leakage current in distribution insulators", IEE Proc. of Generation, Transmission and Distribution, vol. 151, no. 3, pp. 334-
340, 2004.

[5] A. Cavallini, S. Chandrasekar and G. C. Montanari, "Inferring Ceramic Insulator Pollution by an innovative Approach Resorting to PD Detection", IEEE Trans. Dielectrics and Electr. Insul., vol. 14, no. 1, pp. 23-29, 2007.

[6] V. Jayaprakash Narayanan, B. Karthik and S. Chandrasekar, "Flashover Prediction of Polymeric Insulators Using PD Signal Time-Frequency Analysis and BPA Neural Network Technique", Journal of Electrical Engineering \& Technology, vol. 9, no. 4, pp. 1375-1384, 2014.

[7] R. S. Gorur and H. M. Schneider, "Surface resistance measurements on non-ceramic insulators", IEEE Trans. Power Delivery, vol. 16, pp. 801-805, 2001.

[8] Suwarno, "Leakage Current Waveforms of Outdoor Polymeric Insulators and Possibility of Application for Diagnostics of Insulator Conditions", Journal of Electrical Engineering \& Technology, vol. 1, no. 1, pp. 114-119, 2006.

[9] V. Jayaprakash Narayanan, M. Sivakumar, K. Karpagavani and S. Chandrasekar, "Prediction of Flashover andd Pollution Severity of High Voltage Transmission Line Insulators Using Wavelet Transform and Fuzzy C-Means Approach", Journal of Electrical Engineering \& Technology, vol. 9, no. 5, pp. 1677-1685, 2014.

[10] R. Sarathi, S. Chandrasekar and N. Yoshimura, "Investigations into the Surface Condition of the Silicone Rubber Insulation Material using Multiresolution Signal Decomposition", IEEE Trans. Power Delivery, vol. 21, pp. 243-252, 2006.

[11] Shihua Zhao, Xingliang Jiang, Zhijing Zhang, Jianlin $\mathrm{Hu}$, And Lichun Shu, "Flashover voltage prediction of composite insulators based on the characteristics of leakage current", IEEE Trans. Power Delivery, vol. 28, no. 3, pp. 1699-1708, 2013.

[12] R.Sarathi and S.Chandrasekar, "Diagnostic study of the surface condition of the insulation structure using wavelet transform and neural networks", Electric Power Systems Research, Elsevier, vol. 68, pp. 137147, 2004.

[13] S. Kumagai, B. Marungsri, H. Shinokubo, R. Matsuoka and N. Yoshimura, "Comparison of Leakage Current and Aging of Silicone Rubbers and Porcelain in both Field and Salt-fog Tests", IEEE Trans. Dielectrics and Electr. Insul., vol. 13, no. 6, pp. 1286-1302, Dec 2006.

[14] L. H. Meyer, S. H. Jayaram and E. A. Cherney, "Correlation of damage, dry band arcing energy, and temperature in inclined plane testing of silicone rubber for outdoor insulation", IEEE Trans. Dielectrics and Electr. Insul., vol. 11, no. 3, pp. 424-432, 2004.

[15] T.Suda, "Frequency characteristics of leakage current waveforms of an artificially polluted suspension insulator", IEEE Trans. Dielectrics and Electr. Insul., vol. 8, no. 4, pp. 705-709, Aug 2001. 
[16] M. Ugur, D.W. Auckland, B.R. Varlow, and Z. Emin, "Neural Networks to Analyze Surface Tracking on Solid Insulators ", IEEE Trans. Dielectrics and Electr. Insul., vol. 4, no. 6, pp. 763-766, Dec 1997.

[17] S. Chandrasekar, C. Kalaivanan, Andrea Cavallini and Gian Carlo Montanari, "Investigations on Leakage Current and Phase Angle Characteristics of Porcelain and Polymeric Insulator under Contaminated Conditions", IEEE Trans. Dielectr. Electr. Insul., vol. 16, no. 2, pp. 574-583, Apr. 2009.

[18] I.W.C. Lee and P.K. Dash, "S-Transform Based Intelligent System for Classification of Power Quality Disturbance Signals", IEEE Trans. Industrial Electronics, vol. 50, no. 4, pp. 800-805, Aug 2003.

[19] S. Mishra, C.N. Bhende and B.K. Panigrahi, "Detection and Classification of Power Quality Disturbances Using S-Transform and Probabilistic Neural Network", IEEE Trans. on Power Delivery, vol. 23, no. 1, pp. 280-287, Jan 2008.

[20] IEC 60507, Artificial Pollution tests on high voltage insulators to be used on AC systems, 1991.

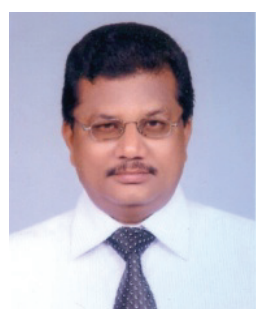

A. Natarajan received the B.E. degree in Electronics and Communication Engineering from College of Engineering, Guindy, Anna University Chennai and M.E. degree in power electronics and drives from Annai Mathammal Sheela Engineering College, Anna University, Coimbatore. He is currently working as an Associate Professor in Annai Mathammal Sheela Engineering College, Namakkal, India. His area of research interest includes applications of signal processing and soft computing techniques for electrical and electronics engineering.

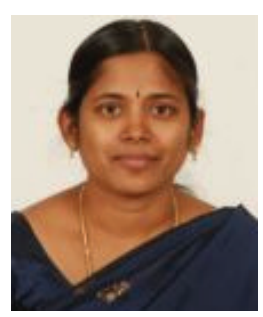

Suthanthiravanitha Narayanan is currently a Professor and Head of the Dept. of EEE in Knowledge Institute of Technology, Salem. She was awarded with Ph.D from Anna University, Chennai in the specialization of Information and Communication Engineering. She also published about 40 journals at International level and 10 journals at National level. She has guided a student for getting internship from IITBombay in the idea of Medical shop explorer in e-yantra IDEAS competition. She is specialized in Biomedical Instrumentation, Embedded Systems, Renewable Energy and Robotics. 